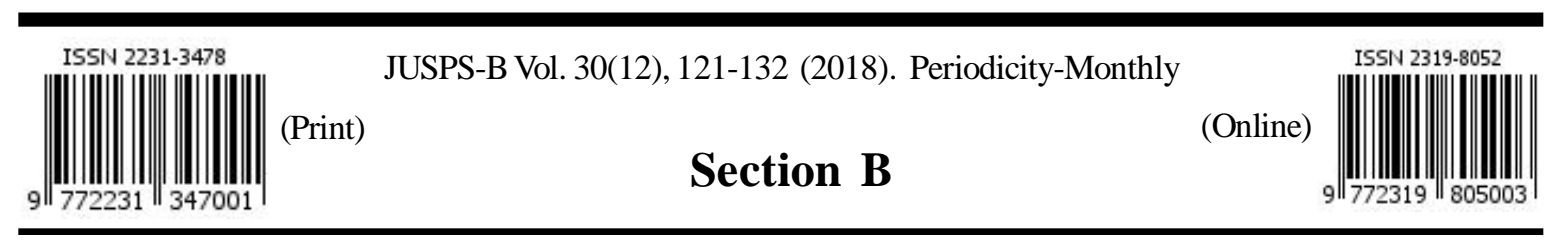

JOURNAL OF ULTRA SCIENTIST OF PHYSICAL SCIENCES
website:- www.ultrascientist.org
Estd. 1989

\title{
Radiation Effects on the Peristaltic flow of a Williamson fluid through a porous medium in a planar channel
}

\author{
B. RANJITHA ${ }^{\mathrm{a}}$ and M. V. SUBBA REDDY ${ }^{\mathrm{bl}}$ \\ ${ }^{a}$ Research Scholar, Department of Mathematics, Rayalaseema University, Kurnool-518002, \\ Andhra Pradesh (India) \\ ${ }^{b}$ Professor, Department of CSE, Sri Venkatesa Perumal College of Engineering \& Technology, \\ Puttur-517583, Andhra Pradesh (India) \\ ${ }^{1}$ Corresponding author: E-mail: drmvsr1979@gmail.com \\ http://dx.doi.org/10.22147/jusps-B/301201
}

Acceptance Date 4th December, 2018, $\quad$ Online Publication Date 17th December, 2018

\begin{abstract}
In this paper, we studied the interaction of heat transfer with peristaltic pumping of a Williamson fluid through a porous medium in a planar channel, under the assumptions of low Reynolds number and long wavelength. The flow is investigated in a wave frame of reference moving with velocity of the wave. The perturbation series in the Weissenberg number $(W e<1)$ was used to obtain explicit forms for velocity field, pressure gradient and friction force per one wavelength. The effects of Weissenberg number We, Darcy number $D a$ and amplitude ratio $\phi$ on the pumping characteristics and heat transfer are discussed through graphs in detail.

Key words : Williamson fluid, Radiation, Peristaltic flow, porous medium.
\end{abstract}

\section{Introduction}

Many researchers considered the fluid to behave like a Newtonian fluid for physiological peristalsis including the flow of blood in arterioles. But such a model cannot be suitable for blood flow unless the nonNewtonian nature of the fluid is included in it. The non-Newtonian peristaltic flow using a constitutive equation for a second order fluid has been investigated by Siddiqui et al ${ }^{13}$ for a planar channel and by Siddiqui and

This is an open access article under the CC BY-NC-SA license (https://creativecommons.org/licenses/by-nc-sa/4.0) 
Schwarz ${ }^{14}$ for an axisymmetric tube. They have performed a perturbation analysis with a wave number, including curvature and inertia effects and have determined range of validity of their perturbation solutions. Subba Reddy et al. ${ }^{16}$ studied the peristaltic flow of a power-law fluid in an asymmetric channel. Peristaltic motion of a Williamson fluid in an asymmetric channel was studied by Nadeem and Akram ${ }^{9}$. Peristaltic pumping of Williamson fluid in a horizontal channel under the effect of magnetic field was investigated by Subba Reddy et al. ${ }^{17}$.

In all the above mentioned studies no porous media has been taken into account. The study of blood flow through arteries are of considerable importance in many cardiovascular diseases particularly arteriosclerosis. In some pathological situations, the distribution of fatty cholesterol and artery clogging blood clots in the lumen of coronary artery can be considered as equivalent to a porous medium. The first study of peristaltic flow through a porous medium is presented by Elsehawey et al. ${ }^{3}$. Elshehawey et al. ${ }^{4}$ have studied the peristaltic motion of a Carreau fluid through a porous medium in a channel. The interaction of peristaltic flow with pulsatile fluid under the effect of a transverse magnetic field through a porous medium bounded by a two-dimensional channel was studied by Afifi and $\mathrm{Gad}^{2}$. Mekheimer and $\mathrm{Arabi}^{7}$ studied the non-linear peristaltic transport of MHD flow through a porous medium. Elshehawey ${ }^{5}$ have studied the peristaltic flow of Newtonian fluid through a porous medium in an asymmetric channel. Navaneeswar Reddy and Viwanatha Reddy ${ }^{11}$ have discussed the slip effects on peristaltic motion of a williamson fluid through a porous medium in a planar channel. Peristaltic flow of phan-thien-tanner fluid in an asymmetric channel with porous medium was discussed by Vajravelu $e t$ $a l .{ }^{19}$. Abdulhadi and Ahmed ${ }^{1}$ have studied the effect of magnetic field on peristaltic flow of Williamson fluid through a porous medium in an inclined tapered asymmetric channel.

The importance of thermodynamic effects of blood in the processes like oxygenation and hemodialysis make the study of heat transfer effects in peristalsis important. Radhakrishnamacharya and Srinivasulu ${ }^{12}$ investigated the interaction of peristalsis and heat transfer. Vajravelu et al. ${ }^{18}$ have investigated the peristaltic flow of a Newtonian fluid through a porous medium in a vertical annulus with heat transfer. Mekheimer Elmaboud ${ }^{8}$ investigated the influence of heat transfer and magnetic field on peristaltic transport of a Newtonian fluid in a vertical annulus. The influence of heat and mass transfer on MHD peristaltic flow through a porous space with compliant walls was investigated by Srinivas and Kothandapani ${ }^{15}$. Hayat et al. ${ }^{6}$ studied the effect of heat transfer on peristaltic flow of an electrically conducting fluid in a porous space. Nadeem and Akram ${ }^{10}$ studied the heat transfer in a peristaltic flow of MHD fluid with partial slip. Vasudev et al. ${ }^{20}$ have investigated the peristaltic pumping of Williamson fluid through a porous medium in a horizontal channel with heat transfer.

In view of these, we studied the interaction of heat transfer with peristaltic pumping of a Williamson fluid through a porous medium in a planar channel, under the assumptions of low Reynolds number and long wavelength. The flow is investigated in a wave frame of reference moving with velocity of the wave. The perturbation series in the Weissenberg number $(W e<1)$ was used to obtain explicit forms for velocity field, pressure gradient and friction force per one wavelength. The effects of Weissenberg number We, Darcy number $D a$ and amplitude ratio $\phi$ on the pumping characteristics and heat transfer are discussed through graphs in detail.

\section{Mathematical Formulation}

We consider the peristaltic motion of a Williamson fluid through a porous medium in a two-dimensional symmetric channel of width $2 a$. The flow is generated by sinusoidal wave trains propagating with constant speed $c$ along the channel walls. A rectangular co-ordinate system $(\mathrm{X}, \mathrm{Y})$ is chosen such that $\mathrm{X}$-axis lies along the centre line of the channel in the direction of wave propagation and Y-axis transverse to it. Since we are 
considering uniform channel therefore the upper wall is maintained at temperature $T_{1}$ and due to symmetry at the center of the channel the change of the temperature taken to be zero (given in Nadeem and Akbar ${ }^{10}$ ). Fig. 1 shows the schematic diagram of the channel.

The wall deformation is given by

$$
Y= \pm H(X, t)= \pm a \pm b \sin \frac{2 \pi}{\lambda}(X-c t)
$$

where $b$ is the amplitude of the wave, $\lambda$ - the wave length and $X$ and $Y$ - the rectangular co-ordinates with $X$ measured along the axis of the channel and $Y$ perpendicular to $X$.

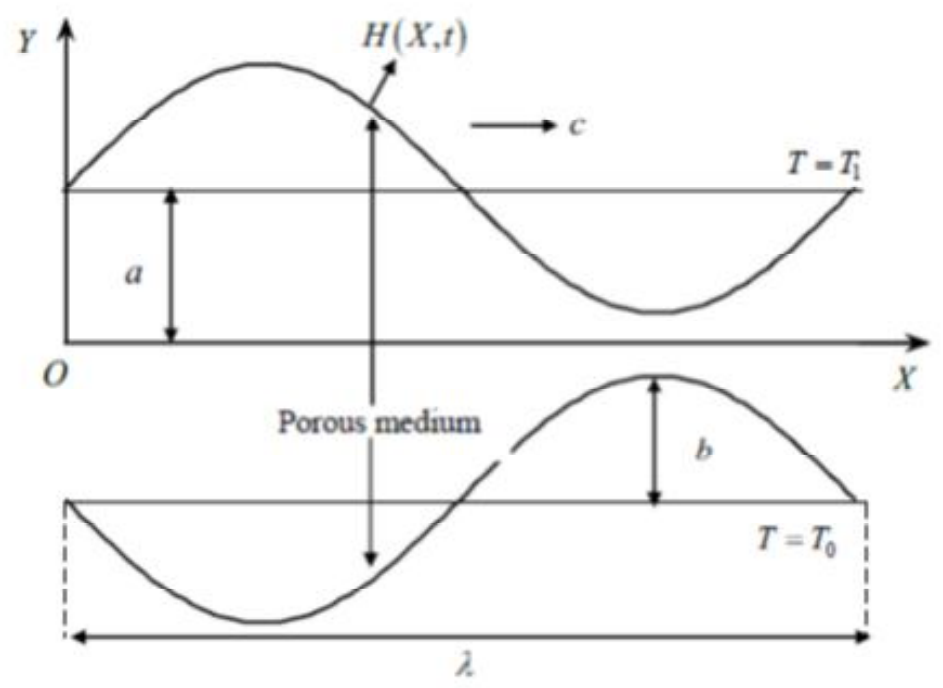

Fig. 1. The Physical Model

The flow is unsteady in the laboratory frame $(X, Y)$. However, in a co-ordinate system moving with the propagation velocity c (wave frame $(x, y)$ ), the boundary shape is stationary. The transformation from fixed frame to wave frame is given by

$$
x=X-c t, y=Y, u=U-c, v=V
$$

where $(u, v)$ and $(U, V)$ are velocity components in the wave and laboratory frames respectively.

The constitutive equation for a Williamson fluid (given in Bird et al.) is

$$
\tau=-\left[\eta_{\infty}+\left(\eta_{0}+\eta_{\infty}\right)(1-\Gamma \dot{\gamma})^{-1}\right] \dot{\gamma}
$$

where $\tau$ is the extra stress tensor, $\eta_{\infty}$ is the infinite shear rate, viscosity $\eta_{0}$ is the zero shear rate viscosity, $\Gamma$ is the time constant and $\dot{\gamma}$ is defined as

$$
\dot{\gamma}=\sqrt{\frac{1}{2}} \sum_{i} \sum_{j} \dot{\gamma}_{i j} \dot{\gamma}_{j i}=\sqrt{\frac{1}{2}} \pi
$$

where $\pi$ is the second invariant stress tensor. We consider in the constitutive Equation (2.3) the case for which $\eta_{\infty}=0$ and $\Gamma \dot{\gamma}<1$ so we can write.

$$
\tau=-\eta_{0}(1+\Gamma \dot{\gamma}) \dot{\gamma}
$$


The above model reduces to Newtonian for $\Gamma=0$

The equations governing the flow in the wave frame of reference are

$\frac{\partial u}{\partial x}+\frac{\partial v}{\partial y}=0$

$\rho\left(u \frac{\partial u}{\partial x}+v \frac{\partial u}{\partial y}\right)=-\frac{\partial p}{\partial x}-\frac{\partial \tau_{x x}}{\partial x}-\frac{\partial \tau_{y x}}{\partial y}-\frac{\mu}{k_{0}}(u+c)$

$\rho\left(u \frac{\partial v}{\partial x}+v \frac{\partial u}{\partial y}\right)=-\frac{\partial p}{\partial y}-\frac{\partial \tau_{x y}}{\partial x}-\frac{\partial \tau_{y y}}{\partial y}-\frac{\mu}{k_{0}} v$

$\zeta\left[u \frac{\partial T}{\partial x}+v \frac{\partial T}{\partial y}\right]=\frac{k}{\rho} \nabla^{2} \mathrm{~T}+v\left\{2\left[\left(\frac{\partial u}{\partial x}\right)^{2}+\left(\frac{\partial v}{\partial y}\right)^{2}\right]+\left(\frac{\partial u}{\partial y}+\frac{\partial v}{\partial x}\right)^{2}\right\}+4 \frac{\alpha^{2}}{\rho c_{\mathrm{p}}}\left(\mathrm{T}-\mathrm{T}_{0}\right)$

where $\rho$ is the density, $\mu$ is the co-efficient of viscosity of the fluid, $k_{0}$ is the permeability of the porous medium, $\zeta$ is the specific heat at constant volume, $v$ is kinematic viscosity of the fluid, $\alpha$ is coefficient of linear thermal expansion of the fluid $k$ is thermal conductivity of the fluid and $T$ is temperature of the fluid.

Introducing the non-dimensional variables defined by

$\bar{x}=\frac{x}{\lambda}, \bar{y}=\frac{y}{a}, \bar{u}=\frac{u}{c}, \bar{v}=\frac{v}{c \delta}, \delta=\frac{a}{\lambda}, \bar{p}=\frac{p a^{2}}{\eta_{0} c \lambda}, h=\frac{H}{a}, \bar{t}=\frac{c t}{\lambda}$,

$\tau_{x x}=\frac{\lambda}{\eta_{0} c} \tau_{x x}, \bar{\tau}_{x y}=\frac{a}{\eta_{0} c} \tau_{x y}, \tau_{y y}=\frac{\lambda}{\eta_{0} c} \tau_{y y}, R e=\frac{\rho a c}{\eta_{0}}, W e=\frac{\Gamma c}{a}$,

$\overline{\dot{\gamma}}=\frac{\dot{\gamma} a}{c}, \bar{q}=\frac{q}{a c}, \theta=\frac{T-T_{0}}{T_{1}-T_{0}}, \operatorname{Pr}=\frac{\rho v \zeta}{k}, E=\frac{c^{2}}{\zeta\left(T_{1}-T_{0}\right)}$

into the Equations (2.6) - (2.9), reduce to (after dropping the bars)

$\frac{\partial u}{\partial x}+\frac{\partial v}{\partial y}=0$

$\operatorname{Re} \delta\left(u \frac{\partial u}{\partial x}+v \frac{\partial u}{\partial y}\right)=-\frac{\partial p}{\partial x}-\delta^{2} \frac{\partial \tau_{x x}}{\partial x}-\frac{\partial \tau_{x y}}{\partial y}-\frac{1}{D a}(u+1)$

$R e \delta^{3}\left(u \frac{\partial v}{\partial x}+v \frac{\partial v}{\partial y}\right)=-\frac{\partial p}{\partial y}-\delta^{2} \frac{\partial \tau_{x y}}{\partial y}-\delta \frac{\partial \tau_{y y}}{\partial y}-\frac{\delta^{2}}{D a} v$

$\operatorname{Re}\left[u \frac{\partial \theta}{\partial x}+v \frac{\partial \theta}{\partial y}\right]=\frac{1}{P r}\left(\delta^{2} \frac{\partial^{2} \theta}{\partial x^{2}}+\frac{\partial^{2} \theta}{\partial y^{2}}\right)+N^{2} \theta+$

$E\left\{4 \delta^{2}\left(\frac{\partial u}{\partial x}\right)^{2}+\left(\frac{\partial u}{\partial y}\right)^{2}+\delta^{4}\left(\frac{\partial v}{\partial x}\right)^{2}+2 \delta^{2} \frac{\partial u}{\partial y} \frac{\partial v}{\partial x}\right\}$

Where

$$
\begin{aligned}
& \tau_{x x}=-2[1+W e \dot{\gamma}] \frac{\partial u}{\partial x} \tau_{x y}=-[1+W e \dot{\gamma}]\left(\frac{\partial u}{\partial x}+\delta^{2} \frac{\partial v}{\partial x}\right), \tau_{y y}=-2 \delta[1+W e \dot{\gamma}] \frac{\partial v}{\partial y} \\
& \dot{\gamma}=\left[2 \delta^{2}\left(\frac{\partial u}{\partial x}\right)^{2}+\left(\frac{\partial u}{\partial y}+\delta^{2} \frac{\partial v}{\partial x}\right)^{2}+2 \delta^{2}\left(\frac{\partial v}{\partial y}\right)^{2}\right]^{\frac{1}{2}},
\end{aligned}
$$


and $D a=\frac{k_{0}}{a^{2}}$ is the Darcy number.

Under lubrication approach, neglecting the terms of order $\delta$ and Re, we get

$$
\begin{aligned}
& \frac{\partial p}{\partial y}=\frac{\partial}{\partial y}\left\{\left[1+W e \frac{\partial u}{\partial y}\right] \frac{\partial u}{\partial y}\right\}-\frac{1}{D a}(u+1) \\
& \frac{\partial p}{\partial y}=0 \\
& \frac{1}{\operatorname{Pr}}\left[\frac{\partial^{2} \theta}{\partial y^{2}}\right]+E\left[\frac{\partial u}{\partial y}\right]^{2}+N^{2} \theta=0
\end{aligned}
$$

From Eq. (2.15) and (2.16), we have

$$
\frac{d p}{d x}=\frac{\partial^{2} u}{\partial y^{2}}+W e \frac{\partial}{\partial y}\left[\left(\frac{\partial u}{\partial y}\right)^{2}\right]-\frac{1}{D a}(u+1)
$$

The corresponding dimensionless boundary conditions are

$$
\begin{aligned}
& \frac{\partial u}{\partial y}=0 \quad \text { at } \quad y=0 \\
& u=-1 \quad \text { at } \quad y=h=1+\phi \cos (2 \pi x) \\
& \frac{\partial \theta}{\partial y}=0 \quad \text { at } \quad y=0 \\
& \theta=1 \quad \text { at } \quad y=h=1+\sin (2 \pi x)
\end{aligned}
$$

The volume flow rate $q$ in a wave frame of reference is given by

$$
q=\int_{0}^{\square} u d y .
$$

The instantaneous flow $\mathrm{Q}(X, t)$ in the laboratory frame is

$$
Q(X, t)=\int_{0}^{h} U d Y=\int_{0}^{h}(u+1) d y=q+h
$$

The time averaged volume flow rate $\bar{Q}$ over one period $T\left(=\frac{\lambda}{c}\right)$ of the peristaltic wave is given by

$$
\bar{Q}=\frac{1}{T} \int_{0}^{T} Q d t=q+1
$$

\section{Solution :}

Since Eq. (2.18) is a non-liner differential equation, it is not possible to obtain closed form solution. Therefore we employ regular perturbation to find the solution.

For perturbation solution, we expand $u, p, q$ and $\theta$ as follows

$$
\begin{aligned}
& u=u_{0}+W e u_{1}+\mathrm{O}\left(W e^{2}\right) \\
& \frac{d p}{d x}=\frac{d p_{0}}{d x}+W e \frac{d p_{1}}{d x}+\mathrm{O}\left(W e^{2}\right) \\
& q=q_{0}+W e q_{1}+\mathrm{O}\left(W e^{2}\right)
\end{aligned}
$$




$$
\theta=\theta_{0}+W e \theta_{1}+\mathrm{O}\left(W e^{2}\right)
$$

Substituting these equations into the Equations (2.17) - (2.22), we obtain

\subsection{System of order $W e^{0}$}

$$
\begin{aligned}
& \frac{d p_{0}}{d x}=\frac{\partial^{2} u_{0}}{\partial y^{2}}-\frac{1}{D a}\left(u_{0}+1\right) \\
& \frac{1}{\operatorname{Pr}}\left[\frac{\partial^{2} \theta_{0}}{\partial y^{2}}\right]+E\left[\frac{\partial u_{0}}{\partial y}\right]^{2}+N^{2} \theta=0
\end{aligned}
$$

and the respective boundary conditions are

$$
\begin{array}{llll}
\frac{\partial u_{0}}{\partial y}=0 & \text { at } & y=0 \\
u_{0}=-1 & \text { at } & y=h \\
\frac{\partial \theta_{0}}{\partial y}=0 & \text { at } & y=0 \\
\theta_{0}=1 & \text { at } & y=h
\end{array}
$$

\subsection{System of order $W e^{1}$}

$$
\begin{aligned}
& \frac{d p_{1}}{d x}=\frac{\partial^{2} u_{1}}{\partial y^{2}}+\frac{\partial}{\partial y}\left[\left(\frac{\partial u_{0}}{\partial y}\right)^{2}\right]-\frac{1}{D a} u_{1} \\
& {\left[\frac{\partial^{2} \theta_{1}}{\partial y^{2}}\right]+N^{2} \operatorname{Pr} \theta_{1}=-\operatorname{PrE} \frac{\partial u_{0}}{\partial y} \frac{\partial u_{1}}{\partial y}}
\end{aligned}
$$

and the respective boundary conditions are

$$
\begin{array}{lll}
\frac{\partial u_{1}}{\partial y}=0 & \text { at } & y=0 \\
u_{1}=0 & \text { at } & y=h \\
\frac{\partial \theta_{1}}{\partial y}=0 & \text { at } & y=0 \\
\theta_{1}=0 & \text { at } & y=h
\end{array}
$$

\subsection{Solution for system of order $W e^{0}$}

Solving Eq. (3.5) using the boundary conditions (3.7) and (3.8), we obtain

$$
u_{0}=D a \frac{d p_{0}}{d x}\left[\frac{\cosh \left(\frac{y}{\sqrt{D a}}\right)}{\cosh \left(\frac{h}{\sqrt{D a}}\right)}\right]-1
$$

Substituting Eq. (3.17) into the Eq. (3.6) and solving the Eq. (3.6), using the boundary conditions (3.9) and (3.10), we obtain

$$
\begin{aligned}
& \theta_{0}=\frac{\cos N \sqrt{P r} y}{\cos N \sqrt{P r} h}\left[1+E P r\left(\frac{D a}{2}\right)\left(\frac{d p_{0}}{d x}\right)^{2} \operatorname{sech}^{2}\left(\frac{h}{\sqrt{D a}}\right)\left(\frac{D a}{4+N^{2} D a P r} \cosh \left(\frac{2 h}{\sqrt{D a}}\right)-\frac{1}{N^{2} P r}\right)\right]- \\
& E P r\left(\frac{D a}{2}\right)^{2}\left(\frac{d p_{0}}{d x}\right)^{2} \operatorname{sech}^{2}\left(\frac{h}{\sqrt{D a}}\right)\left(\frac{D a}{4+N^{2} D a P r} \cosh \left(\frac{2 y}{\sqrt{D a}}\right)-\frac{1}{N^{2} P r}\right)
\end{aligned}
$$


B. Ranjitha, et al., JUSPS-B Vol. 30(12), (2018).

The volume flow rate $q_{0}$ is given by

$$
q_{0}=D a \frac{d p_{0}}{d x}\left(\sqrt{D a} \tanh \left(\frac{h}{\sqrt{D a}}\right)-h\right)-h
$$

From Eq. (3.19), we have

$$
\frac{d p_{0}}{d x}=\frac{\left(q_{0}+h\right)}{D a\left(\sqrt{D a} \tanh \left(\frac{h}{\sqrt{D a}}\right)-h\right)}
$$

\subsection{Solution for system of order $W e^{1}$}

Substituting Equation (3.17) in the Eq. (3.11) and solving the Eq. (3.11), using the boundary conditions (3.13) and (3.14), we obtain

$$
u_{1}=D a \frac{d p_{1}}{d x}\left[\frac{\cosh \left(\frac{y}{\sqrt{D a}}\right)}{\cosh \left(\frac{h}{\sqrt{D a}}\right)}-1\right]+\frac{1}{3} \frac{(D a)^{\frac{3}{2}}\left(\frac{d p_{0}}{d x}\right)^{2}}{\cosh ^{2}\left(\frac{h}{\sqrt{D a}}\right)}\left[\begin{array}{c}
2 \sinh \left(\frac{y}{\sqrt{D a}}\right)-\sinh \left(\frac{2 y}{\sqrt{D a}}\right) \\
+A_{1} \cosh \left(\frac{y}{\sqrt{D a}}\right)
\end{array}\right]
$$

where $A_{1}=2\left[\cosh \left(\frac{h}{\sqrt{D a}}\right)-1\right] \tanh \left(\frac{h}{\sqrt{D a}}\right)$.

Substituting Equations (3.17) and (3.21) into the Eq. (3.12) and solving the Eq. (3.12), using the boundary conditions (3.15) and (3.16), we get

$$
\begin{aligned}
& \theta_{1}=\frac{d P_{0}}{d x} \frac{\cos N \sqrt{\operatorname{Pr}} y}{\cos N \sqrt{\operatorname{Pr}} h}\left[\frac{d P_{1}}{d x} A_{3}+\left(\frac{d P_{0}}{d x}\right)^{2}\left(A_{4}-A_{2} \sin N \sqrt{\operatorname{Pr}} h\right)\right]+\left(\frac{d P_{0}}{d x}\right)^{2} A_{2} \sin N \sqrt{\operatorname{Pr} h} \\
& {\left[\frac{1}{2}(D a)^{2} \frac{d P_{0}}{d x} \frac{d P_{1}}{d x} \frac{1}{4+N^{2} D a P r} \frac{\cosh \left(\frac{2 y}{\sqrt{D a}}\right)}{\cosh ^{2}\left(\frac{h}{\sqrt{D a}}\right)}+\frac{1}{2 P r}(D a)^{2} \frac{d P_{0}}{d x} \frac{d P_{1}}{d x} \frac{1}{\cosh ^{2}\left(\frac{h}{\sqrt{D a}}\right)}\right.} \\
& \operatorname{PrE}+\frac{2}{3}(D a)^{\frac{5}{2}}\left(\frac{d P_{0}}{d x}\right)^{3} \frac{\sinh \left(\frac{2 y}{\sqrt{D a}}\right)}{\cosh ^{3}\left(\frac{h}{\sqrt{D a}}\right)}-\frac{1}{3}(D a)^{\frac{5}{2}}\left(\frac{d P_{0}}{d x}\right)^{3} \frac{1}{9+N^{2} D a P r} \frac{\sinh \left(\frac{3 y}{\sqrt{D a}}\right)}{\cosh ^{3}\left(\frac{h}{\sqrt{D a}}\right)} \\
& +\frac{1}{3}(D a)^{\frac{5}{2}}\left(\frac{d P_{0}}{d x}\right)^{3} \frac{1}{1+N^{2} D a P r} \frac{\sin \square\left(\frac{y}{\sqrt{D a}}\right)}{\cosh ^{3}\left(\frac{h}{\sqrt{D a}}\right)}+\frac{A_{1}}{6}(D a)^{\frac{5}{2}}\left(\frac{d P_{0}}{d x}\right)^{3} \frac{1}{4+N^{2} D a P r} \frac{\cosh \left(\frac{2 y}{\sqrt{D a}}\right)}{\cosh ^{3}\left(\frac{h}{\sqrt{D a}}\right)} \\
& -\frac{A_{1}}{6 \operatorname{Pr} N^{2}}(D a)^{\frac{5}{2}}\left(\frac{d P_{0}}{d x}\right)^{3} \frac{1}{\cosh ^{3}\left(\frac{h}{\sqrt{D a}}\right)} \\
& A_{2}=\frac{E \sqrt{P r}}{N}(D a)^{2} \frac{1}{\cosh ^{3}\left(\frac{h}{\sqrt{D a}}\right)}\left[\frac{2}{3} \frac{1}{4+N^{2} D a P r}-\frac{1}{9+N^{2} D a P r}+\frac{1}{3} \frac{1}{3+N^{2} D a P r}\right]
\end{aligned}
$$


$A_{3}=\frac{\operatorname{Pr} E}{2} D a \frac{1}{\cosh \left(\frac{h}{\sqrt{D a}}\right)}\left[D a \frac{1}{4+N^{2} D a P r}+\frac{1}{\cosh \left(\frac{h}{\sqrt{D a}}\right)} \frac{1}{N^{2} P r}\right]$

and

$$
\begin{aligned}
A_{4}=\frac{P r E}{3}(D a)^{\frac{3}{2}} & \frac{1}{\cosh ^{2}\left(\frac{h}{\sqrt{D a}}\right)}\left[\frac{D a}{4+N^{2} D a P r} \sinh \left(\frac{2 h}{\sqrt{D a}}\right)-\frac{D a}{9+N^{2} D a P r} \sinh \left(\frac{3 h}{\sqrt{D a}}\right)\right. \\
+ & \left.\frac{D a}{4+N^{2} D a P r} \sinh \left(\frac{2 h}{\sqrt{D a}}\right)+\frac{A_{1}}{2} \frac{D a}{4+N^{2} D a P r} \cosh \left(\frac{2 h}{\sqrt{D a}}\right)-\frac{A_{1}}{2 N^{2} P r}\right]
\end{aligned}
$$

The volume flow rate is given by

$$
q_{1}=D a \frac{d p_{1}}{d x}\left[\sqrt{D a} \tanh \left(\frac{h}{\sqrt{D a}}\right)-h\right]+\frac{(D a)^{\frac{3}{2}}\left(\frac{d p_{0}}{d x}\right)^{2}}{3 \cosh ^{2}\left(\frac{h}{\sqrt{D a}}\right)} A_{5}
$$

where

$A_{5}=2 \sqrt{D a}\left(\cosh \left(\frac{h}{\sqrt{D a}}\right)-1\right)+A_{1} \sinh \left(\frac{h}{\sqrt{D a}}\right)-\frac{\sqrt{D a}}{2}\left[\cosh \left(\frac{2 h}{\sqrt{D a}}\right)-1\right]$.

From Equations (3.23) and (3.20), we have

$$
\frac{d p_{1}}{d x}=\frac{q_{1}}{D a\left[\sqrt{D a} \tanh \left(\frac{h}{\sqrt{D a}}\right)-h\right]}-\frac{(D a)^{\frac{3}{2}} A_{5}\left(q_{0}+h\right)^{2}}{3\left(D a\left[\sqrt{D a} \tanh \left(\frac{h}{\sqrt{D a}}\right)-h\right]\right)^{3} \cosh ^{2}\left(\frac{h}{\sqrt{D a}}\right)}
$$

Substituting Equations (3.20) and (3.24) into the equation Eq. (3.27), we get

$$
\frac{d p}{d x}=\frac{(q+h)}{\operatorname{Da}\left[\sqrt{\operatorname{Da} t a n h}\left(\frac{h}{\sqrt{D a}}\right)-h\right]}-W e \frac{A_{5}\left(q_{0}+h\right)^{2}}{3(D a)^{\frac{3}{2}}\left(\operatorname{Da}\left[\sqrt{\operatorname{Da} t a n h}\left(\frac{h}{\sqrt{D a}}\right)-h\right]\right)^{3} \cosh ^{2}\left(\frac{h}{\sqrt{D a}}\right)}
$$

The dimensionless pressure rise per one wavelength in the wave frame is defined as

$$
\Delta P=\int_{0}^{1} \frac{d p}{d x} d x
$$

As $\mathrm{N} \rightarrow 0$ our results coincides with the results of vasudev et $a l .{ }^{20}$.

\section{Discussion of the results}

In Fig 2, shows the variation of pressure gradient $\frac{d p}{d x}$ for different values We with $\phi=0.5 D a=0.1$ and $q=-1$. It is observed that the variation of axial pressure gradient $\frac{d p}{d x}$ increases with increasing $W e$.

In Fig 3, depicts the variation of pressure gradient $\frac{d p}{d x}$ for different values $D a$ with $\phi=0.6$ and 
$W e=0.01$. It is noted that the variation of axial pressure gradient $\frac{d p}{d x}$ decreases with increasing $D a$.

In Fig 4, shows the variation of axial pressure gradient $\frac{d p}{d x}$ for different values of $\phi$ with $W e=0.01$ and $D a=0.1$. It is found that the variation of axial pressure gradient $\frac{d p}{d x}$ increases for different values of $\phi$ except at the end of the channel.

In Fig 5, presents the variation of pressure rise $\Delta p$ with $\bar{Q}$ for different values of We with $\phi=0.5$ and $D a=0.1$. It is observed that the average volume flow rate $\bar{Q}$ increases in the pumping $(\Delta p<0)$ and free-pumping $(\Delta p=0)$ regions with increasing $W e$, while it increases in the co-pumping region with increasing $W e$ for chosen $(\Delta p<0)$.

In Fig 6, shows the variation of pressure rise $\Delta p$ with $\bar{Q}$ for different values of Darcy number $D a$ with $\phi=0.5$ and $W e=0.1$. It is found that any two curves intersecting in first quadrant to the left of the point of intersection, the $\bar{Q}$ decreases with increasing $D a$ whereas to the right of this point of intersection $\bar{Q}$ increasing with increasing $D a$.

In Fig 7, presents the variation of pressure rise $\Delta p$ with $\bar{Q}$ for different values of amplitude ratio $\phi$ with $W e=0.01$ and $D a=0.1$. It is observed that the $\bar{Q}$ increases with increasing $\phi$ both in the pumping and freepumping regions, whereas it decreases with increasing $\phi$ in the co-pumping region for chosen $(\Delta p<0)$

In Fig 8 shows the temperature profiles for different values of We with $\phi=0.5, D a=0.1, \operatorname{Pr} E=1, N=1$, $x=0.1, q_{0}=-1, q_{1}=0$. It is noted that the temperature $\theta$ increases with increasing We.

In Fig 9 shows the temperature profiles for different values of Darcy number $D a$ with $\phi=0.5, W e=0.01$, $\operatorname{Pr} E=1, N=1, x=0.1, q_{0}=-1, q_{1}=0$. It is found that the temperature $\theta$ increases with increasing $D a$.

In Fig 10 shows the temperature profiles for different values of Amplitude ratio $\phi$ with $D a=0.1$, We $=$ $0.01, \operatorname{Pr} E=1, N=1, x=0.2, q_{0}=-1, q_{1}=0$. It is found that the temperature $\theta$ increases with increasing $\phi$.

In Fig 11 shows the temperature profiles for different values of $\operatorname{PrE}$ with $\operatorname{Da}=0.1$, $W e=0.01, \phi=0.5, x=0.1, N=1, q_{0}=-1, q_{1}=0$. It is observe that the temperature $\theta$ increases with increasing $\operatorname{PrE}$.

In Fig 12 shows the temperature profiles for different values of $N$ with $D a=0.1, W e=0.01, \phi=0.5, \operatorname{Pr} E=1$, $x=0.1, q_{0}=-1, q_{1}=0$. It is found that the temperature $\theta$ increases with increasing $N$.

\section{Conclusions}

In this paper, we studied the radiation Effects on the Peristaltic flow of a Williamson fluid through a porous medium in a planar channel, under the assumptions of low Reynolds number and long wavelength. A closed form solutions are obtained for the velocity, temperature and the pressure gradient. It is observed that, the axial pressure gradient and the time averaged volume flow rate increases with increasing $W e$ and $\phi$, while they decreases with $D a$. The temperature increases with increasing the parameters $W e, D a, P r, \phi, N$.

\section{Scope of future work :}

This paper can be extended by considering slip effects on the peristaltic flow of a Williamson fluid through porous medium with radiation. 


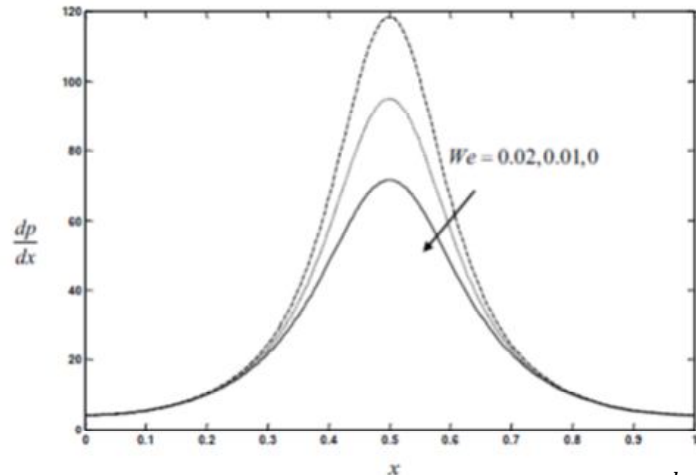

Fig. 2 The variation of axial pressure gradient $\frac{d p}{d x}$ for different values of We with $\phi=0.5$ $D a=0.1$ and $q=-1$.

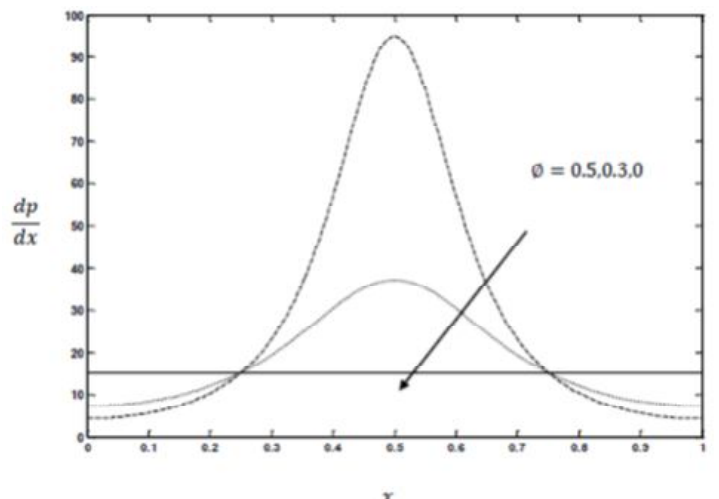

Fig. 4 The variation of axial pressure gradient $\frac{d p}{d x}$ for different values of $\phi$ with $W e=0.01$ and $D a=0.1$.

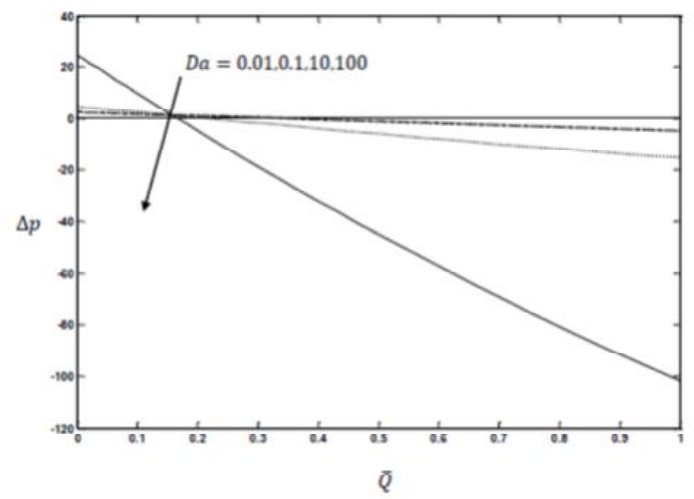

Fig. 6 The variation of pressure rise $\Delta p$ with $\bar{Q}$ for different values of Darcy number $D a$ with $\phi=0.6$ and $W e=0.01$.

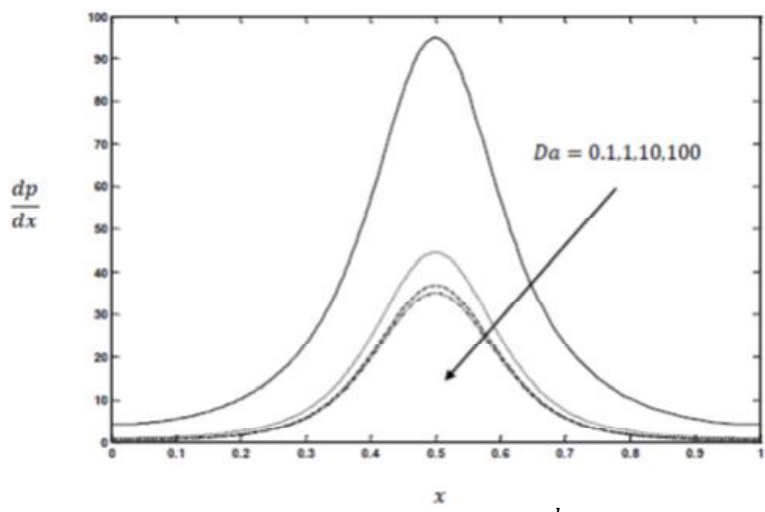

Fig. 3 The variation of pressure rise $\frac{d p}{d x}$ for different values of $D a$ with $\phi=0.6$ and $W e=0.01$.

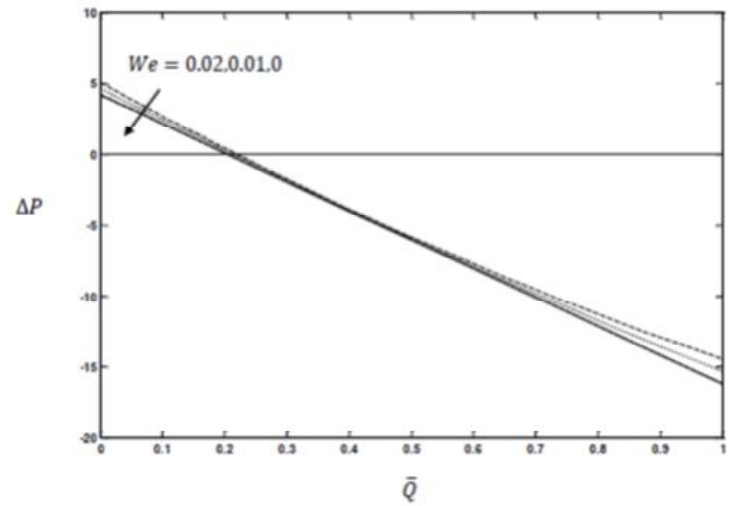

Fig. 5 The variation of pressure rise $\Delta p$ with $\bar{Q}$ for different values of We with $\phi=0.5$ and $D a=0.1$.

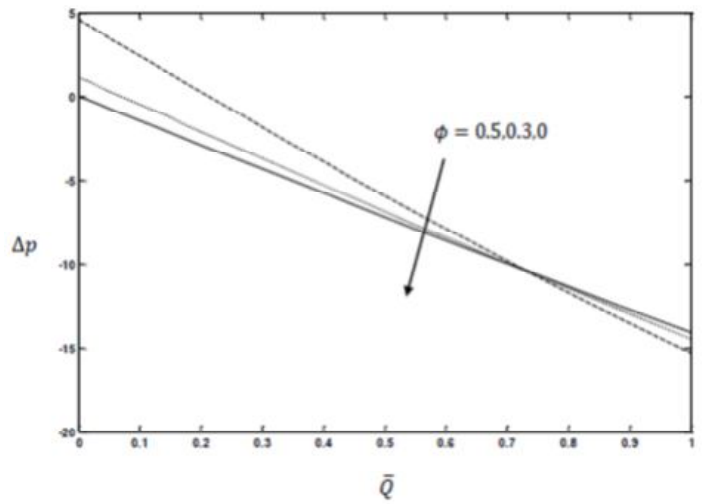

Fig. 7 The variation of pressure rise $\Delta p$ with $\bar{Q}$ for different values of amplitude ratio $\phi$ with $W e=0.01$ and $D a=0.1$. 


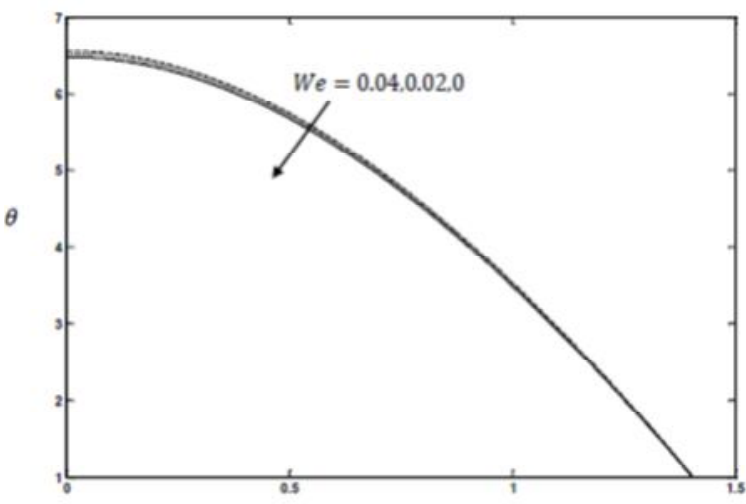

$y$

Fig. 8 Temperature profiles for different values of We with $\phi=0.5, D a=0.1, \operatorname{Pr} E=1, N=1, x=0.1$,

$$
q_{0}=-1, q_{1}=0
$$

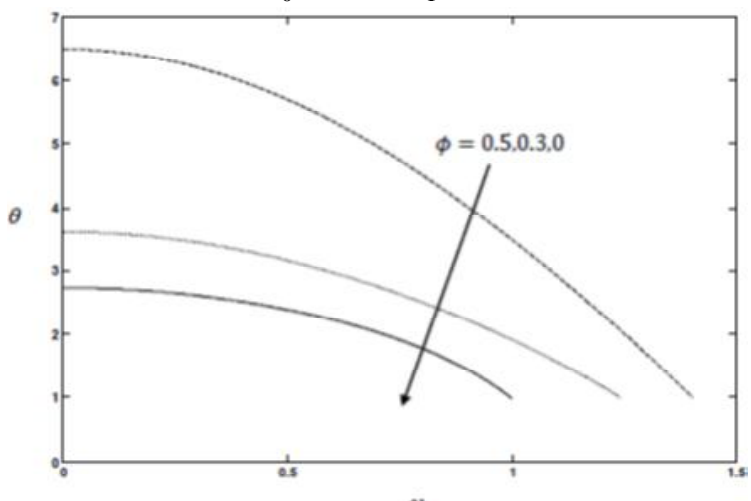

Fig. 10 Temperature profiles for different values of amplitude ratio $\phi$ with $W e=0.01, D a=0.1$, $\operatorname{Pr}=1, E=1, x=0.2, q_{0}=-1, q_{1}=0$

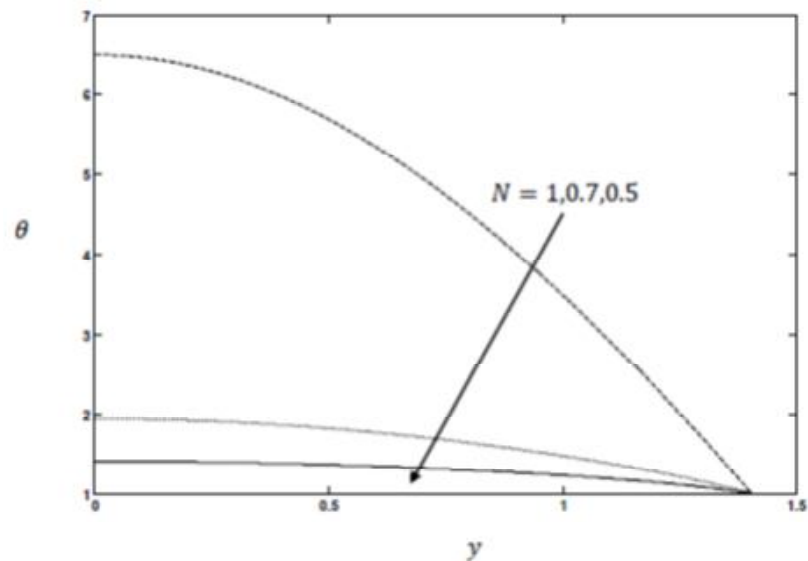

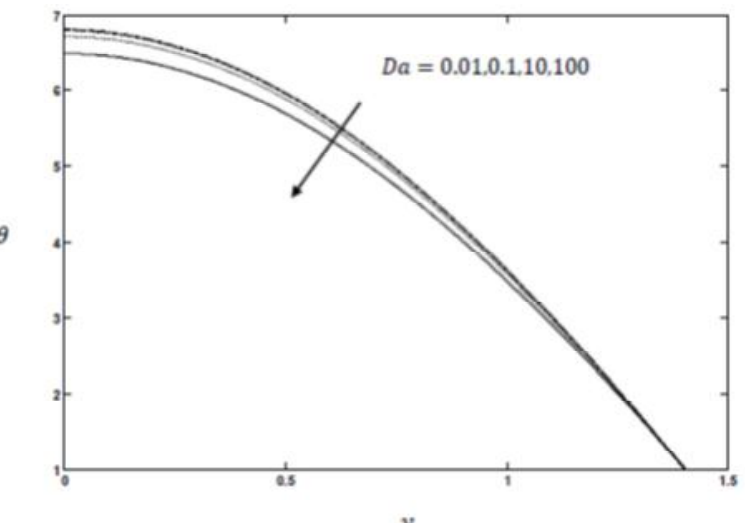

$y$

Fig. 9 Temperature profiles for different values of Darcy number $D a$ with $\phi=0.5, W e=0.01$, $\operatorname{Pr}=1, E=1, x=0.1, q_{0}=-1, q_{1}=0$

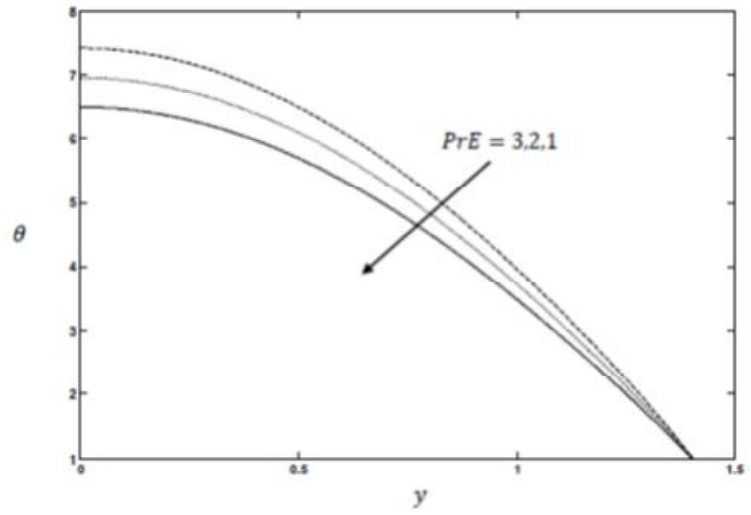

Fig. 11 Temperature profiles for different values of $\operatorname{Pr} E$ with $\phi=0.5, W e=0.01, D a=0.1, x=0.1$, $q_{0}=-1, q_{1}=0$

Fig. 12 Temperature profiles for different values of $N$ with $\phi=0.5$, $W e=0.01, D a=0.1, \operatorname{Pr} E=1$, $x=0.1, q_{0}=-1, q_{1}=0$ 
References

1. Abdulhadi, A. M. and Ahmed, T. S., Effect of magnetic field on peristaltic flow of Williamson fluid through a porous medium in an inclined tapered asymmetric channel, Mathematical Theory and Modeling, Vol. 7, pp. 549-65, (2017).

2. Afifi, N.A.S. and Gad, N.S. Interaction of peristaltic flow with pulsatile magneto fluid through a porous medium, Acta Mechanica, 149, pp. 229-237. (2001).

3. El Shehawey, E.F., Mekheimer, Kh. S., Kaldas, S. F. and Afifi, N. A. S. Peristaltic transport through a porous medium, J. Biomath., 14, pp. 425-431,(1999).

4. El Shehawey, E.F., Sobh, A.M.F. and Elbarbary, E.M.E. Peristaltic motion of a generalized Newtonian fluid through a porous medium, Phys. Soc. Jpn., 69, pp.401-407, (2000).

5. El Shehawey, E.F., Eldabe, N.T., Elghazy, E.M. and Ebaid, A. peristaltic transport in an asymmetric channel through a porous medium, Appl. Math. Comput. 182, pp. 140-150, (2006).

6. Hayat, T., Umar Qureshi, M. and Hussain, Q. Effect of heat transfer on the peristaltic flow of an electrically conducting fluid in a porous space, Applied Mathematical Modelling 33, pp. 1862-1873, (2009).

7. Mekheimer, Kh.S. and Al-Arabi, T.H. Nonlinear peristaltic transport of MHD flow through a porous medium, Int. J. Math. Math. Sci., 26, pp. 1663-1682, (2003).

8. Mekheimer, Kh. S. and Abd elmaboud, Y. The influence of heat transfer and magnetic field on peristaltic transport of a Newtonian fluid in a vertical annulus: application of an endoscope, Phys. Lett. A 372, pp. 1657-1665, (2008).

9. Nadeem, S. and Akram, S. Peristaltic flow of a Williamson fluid in an asymmetric channel, Comm. Non linear sic. Numer. Simul., 15, pp. 1705-1716, (2010).

10. Nadeem, S. and Akram, S. Heat transfer in a peristaltic flow of MHD fluid with partial slip, Commun. Nonlinear Sci. Numer. Simul. 15, pp. 312-321, (2010).

11. Navaneeswara Reddy, S. and Viswanatha Reddy, G. Slip effects on peristaltic motion of a williamson fluid through a porous medium in a planar channel, Asian Journal of Current Engineering and Maths 2: 1 Jan -Feb, pp. 53-58, (2013).

12. Radhakrishnamacharya, G. and Srinivasulu, Ch. Influence of wall properties on peristaltic transport with heat transfer, C. R. Mecanique, 335, pp. 369-373, (2007).

13. Siddiqui, A.M., Provost, A. and Schwarz, W.H. Peristaltic pumping of a second-order fluid in a planar channel, Rheol.Acta, 30, pp. 249-262, (1991).

14. Siddiqui, A.M. and Schwarz, W.H. Peristaltic flow of a second order fluid in tubes, J. Non-Newtonian Fluid Mech., 53, pp. 257-284, (1994).

15. Srinivas, S. and Kothandapani, M. The influence of heat and mass transfer on MHD peristaltic flow through a porous space with compliant walls, Appl. Math. Comput. 213, pp. 197-208, (2009).

16. Subba Reddy, M.V., Ramachandra Rao, A. and Sreenadh, S. Peristaltic motion of a power law fluid in an asymmetric channel, Int. J. Non-Linear Mech., 42, pp. 1153 -1161,(2007).

17. Subba Reddy, M.V., Jayarami Reddy, B. and Prasanth Reddy D. Peristaltic pumping of Williamson fluid in a horizontal channel under the effect of magnetic field. International Journal of Fluid Mechanics 3, pp. 89-109. (2011).

18. Vajravelu, K., Radhakrishnamacharya, G. and Radhakrishnamurty V. Peristaltic flow and heat transfer in a vertical porous annulus with long wave approximation. Int J Nonlinear Mech, 42, pp. 754 -759, (2007).

19. Vajravelu, K., Sreenadh, S., Lakshminarayana, P., Sucharitha, G. and Rashidi, M. M. Peristaltic flow of phan-thien-tanner fluid in an asymmetric channel with porous medium, Journal of Applied Fluid Mechanics, Vol. 9, No. 4, pp. 1615-1625, (2016).

20. Vasudev, C., Rajeswara Rao, U., Subba Reddy, M.V. and Prabhakar Rao, G. Peristaltic Pumping of Williamson fluid through a porous medium in a horizontal channel with heat transfer, Am. J. Sci. Ind. Res., 1(3), pp. 656666 (2010). 